\title{
Profile of apalutamide in the treatment of metastatic castration-resistant prostate cancer: evidence to date
}

This article was published in the following Dove Press journal:

OncoTargets and Therapy

\section{Julio T Chong' \\ William K Oh${ }^{2}$ \\ Bobby C Liaw ${ }^{2}$}

'Department of Urology, Icahn School of Medicine at Mount Sinai, New York, NY, USA; ${ }^{2}$ Department of Medicine, Division of Hematology and Medical Oncology, Icahn School of Medicine at Mount Sinai, New York, NY, USA
Correspondence: Julio T Chong Icahn School of Medicine at Mount Sinai, I Gustave L. Levy Place, New York, NY 10029, USA

$\mathrm{Tel}+\mathrm{I} 21224 \mid 6500$

Fax + I 2128763246

Email julio.chong@mountsinai.org

\begin{abstract}
Advances in therapies have led to the approval of six therapeutic agents since 2004, each demonstrating overall survival benefit in randomized studies, and these have significantly improved the outlook for men facing metastatic castration-resistant prostate cancer (CRPC). More recently, efforts have been directed at trying to effect change at earlier phases of the disease. Apalutamide (ARN-509), a second-generation androgen receptor antagonist, recently received approval in the nonmetastatic (M0) CRPC space. Similar to enzalutamide, apalutamide inhibits the binding of androgen to androgen receptor (AR), nuclear translocation of the androgen-AR complex, and binding of AR transcription complex to DNA-binding sites and transcription elements. Phase I and II trial experience demonstrates the safety and tolerability of apalutamide, as well as its efficacy in effecting prostate-specific antigen response and radiographic-free survival in CRPC. US Food and Drug Administration approval in M0 CRPC was granted following positive results from the phase III SPARTAN study, where apalutamide demonstrated significant improvements in metastasis-free survival and time to symptomatic progression as compared to placebo.
\end{abstract}

Keywords: antiandrogens, castration-resistant prostatic cancer, investigational new drugs

\section{Introduction}

Prostate cancer is the second most commonly diagnosed solid malignancy in men in the United States, accounting for approximately 26,730 deaths in 2017, with the majority of these deaths due to metastatic castration-resistant prostate cancer (mCRPC). This makes prostate cancer the third most lethal cause of cancer-related deaths in American men. ${ }^{1}$ The initial approach for localized prostate cancer is with curative intent; treatment modalities include radiation therapy and radical prostatectomy. However, for the proportion of men who unfortunately present with de novo metastatic disease, or develop metastatic recurrence after initial localized therapy, the disease ultimately evolves to become castration resistant, which is associated with a median time of death of approximately 30 months. $^{2}$

Androgen deprivation therapy (ADT) has remained a mainstay of treatment for metastatic disease, affecting regression of disease and alleviation of symptoms in most men, but there is inevitably acquired prostate cancer resistance to ADT. Mechanisms of resistance include autogenous androgen biosynthesis by the cancer cells, androgen receptor (AR) gene amplification, AR protein overexpression, and AR gene mutations. ${ }^{3}$ Second-generation AR antagonists have been developed in recent years, including the US Food and Drug Administration (FDA)-approved enzalutamide (Xtandi, Astellas, 
Northbrook, IL, USA). This paper details the profile of another second-generation AR antagonist, apalutamide (ARN-509), which will play an important role in the future treatment landscape of mCRPC.

\section{AR antagonism: mechanisms of action}

The AR plays a crucial role in the pathogenesis of the disease and remains a key therapeutic target even in castration resistant disease. Testosterone and its metabolite, dihydrotestosterone (DHT) are the two principal androgens responsible for the growth of the prostate gland. The $A R$ gene is located on chromosome Xq11-q13 and consists of eight exons contributing to a central DNA-binding domain, an N-terminal transactivation domain, and a C-terminal ligand-binding domain on which androgens act. ${ }^{4-6}$ When androgens bind to the AR, conformational changes in the AR allow the androgen-AR complex to form a homodimer, translocate to the nucleus, bind to specific DNA sequences, and induce transcription of related genes. ${ }^{6-8}$

The understanding that androgen deprivation is an effective treatment in metastatic prostate cancer was first demonstrated by Huggins and Hodges ${ }^{9}$ in 1941. As research into the role of the AR continued, mechanisms were better understood and drugs targeting AR were developed. AR antagonists work by competitively and preferentially binding to the AR and blocking the binding of endogenous androgens such as testosterone and DHT and interrupting the androgen dependent cellular cascade that leads to prostate cancer growth.

\section{Apalutamide in contrast to bicalutamide and enzalutamide}

While initial studies of AR antagonists were more focused on flutamide and nilutamide, most subsequent studies evaluated bicalutamide, which remains the most widely used first-generation antiandrogen. ${ }^{6}$ Bicalutamide can function as an AR agonist in castration-resistant states by undergoing an antagonist-to-agonist switch. ${ }^{10,11}$ This is most notably demonstrated by the antiandrogen withdrawal syndrome, which consists of prostate-specific antigen (PSA) reduction and clinical improvement of symptoms upon bicalutamide discontinuation. ${ }^{12}$

Enzalutamide is a second-generation AR antagonist with additional mechanisms of actions beyond the first-generation agents. Not only does it inhibit binding of androgens to the $\mathrm{AR}$, it also prevents androgen-AR complex nuclear translocation and inhibits androgen-AR complex binding to DNA response elements. ${ }^{13}$ Additionally, enzalutamide does not appear to exhibit an antagonist-to-agonist switch in a small cohort of patients upon withdrawal of enzalutamide. ${ }^{14}$ Schrader et $\mathrm{l}^{15}$ suggests that the PSA rise with withdrawal of enzalutamide may have been measured too soon in von Klot et al's study ${ }^{14}$ and that there may be residual enzalutamide masking the full effect. Moreover, since other mutants can induce antagonist-to-agonist switch in second-generation antiandrogens, enzalutamide may not be entirely immune to the switch. ${ }^{15}$ The safety and efficacy profile of enzalutamide have been evaluated in two placebo-controlled, multicenter phase III trials (AFFIRM and PREVAIL), which led to its approval in mCRPC in 2012., ${ }^{2,16}$ Enzalutamide is primarily hepatically eliminated with a half-life of 5.8 days and reaches a steady state in 28-days, ${ }^{16}$ with its most common adverse events being fatigue, back pain, hot flushes, hypertension, and diarrhea. ${ }^{11}$ Notably, in the initial studies of enzalutamide, five seizures were reported in the AFFIRM trial, one in the PREVAIL trial, and two in the TERRAIN trial, 2,12,14,16 leading to its contraindication in patients with history of seizures, or at risk of seizures. The mechanism of action is likely secondary to antagonism of the central nervous system GABA $_{\mathrm{A}}$ receptors. $^{17}$

Apalutamide is a synthetic biaryl thiohydantoin compound that was discovered with structure-activity relationship medicinal chemistry studies. ${ }^{10}$ In vivo and animal studies of apalutamide were first reported by Clegg et al, ${ }^{11}$ which demonstrated the safety and efficacy of the drug. It binds to the same ligand-binding site as bicalutamide but has a sevento ten-fold increased affinity for the AR receptor. Apalutamide was initially tested against the LNCaP/AR prostate cancer cell line, which was made to overexpress AR two- to three-fold above wild type, consistent with metastatic prostate cancer. It did not exhibit agonist activity on AR even at high concentrations of $10 \mu \mathrm{mol} / \mathrm{L}$. To determine if apalutamide similarly inhibits nuclear translocation, Clegg et al ${ }^{11}$ used lysed treated AR-EYFP cell lines to determine percentages of androgen-AR complex located within cytoplasmic and or nuclear compartments. In apalutamide-treated cells, it was seen that the expression of the fluorescent AR-EYFP fusion protein complex was primarily located within the cytoplasmic compartment, as opposed to control and bicalutamide treated cells. To determine if apalutamide could prevent androgen-AR complex binding to DNA response elements for the proportion that did make it to the nucleus, chromatin immunoprecipitation experiments were run with the $\mathrm{LNCaP} /$ AR cell lines. The studies showed that apalutamide-treated cells were able to prevent AR-complex binding to the PSA and TMPSS 2 genes. In vivo efficacy of apalutamide was assessed using castrate immunodeficient mice harboring 
LNCaP/AR xenograft tumors. With bicalutamide-treated mice, only one in ten mice showed $\geq 50 \%$ regression of tumors as opposed to apalutamide-treated mice, where eight of ten mice showing $\geq 50 \%$ tumor regression. ${ }^{11}$

Head-to-head comparison of apalutamide and enzalutamide was made in the same castrate immunodeficient mice bearing the $\mathrm{LNCaP} / \mathrm{AR}$ prostate cancer cell line using dosages of 1,10 , or $30 \mathrm{mg} / \mathrm{kg} / \mathrm{d}$. Thirteen out of 20 mice treated with apalutamide treated at $30 \mathrm{mg} / \mathrm{kg} / \mathrm{d}$ had $\geq 50 \%$ regression of tumor compared to $3 / 19$ mice treated with enzalutamide. Finally, dosages needed to achieve steady-state plasma concentrations of apalutamide were two- to four-fold lower than enzalutamide with similar intratumoral levels, suggesting that apalutamide had higher tumor/plasma ratios, indicating that apalutamide may reach therapeutic levels at lower concentrations, allowing for a greater potential for dose escalation. Central nervous system levels of apalutamide are also twofold lower than that of enzalutamide, suggesting a reduced risk of seizure activity and central nervous system toxicity. ${ }^{11}$ Although the kinetics of enzalutamide and apalutamide have not been directly compared in humans, only inferred, similar tolerability of the two agents in mouse models even at high concentrations has been well documented with the exception that apalutamide's optimal biological dose may be lower, lying between 10 and $30 \mathrm{mg} / \mathrm{kg} / \mathrm{d}$ for apalutamide and $30-100 \mathrm{mg} / \mathrm{kg} / \mathrm{d}$ for enzalutamide. ${ }^{11}$ Finally, the halflives, time to achieve steady-state concentrations and initial maximum tolerable dose are similar between enzalutamide and apalutamide in subsequent human studies, suggesting that the earlier mouse models translated similarly to human studies. ${ }^{11,17,18}$

\section{Phase I study of apalutamide}

Due to the impressive results of apalutamide to reduce tumor size in both castrate-sensitive and -resistant mouse models, and its higher therapeutic index, apalutamide proceeded to
Phase I clinical testing to determine its pharmacokinetics and safety, in order to recommend an efficacious dose for Phase II studies. ${ }^{11,19}$ Men with mCRPC with either three consecutive rising PSA levels with the minimum PSA at time of study at $2 \mathrm{ng} / \mathrm{mL}$, or evidence of soft tissue or bone disease but without brain metastases, were recruited for the study and assigned to escalating doses of apalutamide, starting at $30 \mathrm{mg}$. Dose-limiting toxicity (DLT) was measured in three patients for each dose, up to 28 days. Blood samples were obtained from 0.5 hours up to 96 hours after apalutamide dosing for pharmacokinetic studies. All patients were offered positron emission tomography/computed tomography with 16B-[18F] fluoro-alpha-dihydrotestosterone (FDHT) scans; uptake of FDHT reflected AR expression and as such, reduction in FDHT uptake indicated adequate inhibition blockade of the AR. PSA changes were determined by percent change at 12 weeks and changes in radiographic imaging were determined according to Response Evaluation Criteria in Solid Tumors guidelines and radionuclide bone scans. ${ }^{19}$

Peak plasma concentrations of apalutamide occurred after 2-3 hours after oral administration with a mean halflife of 3-4 days. Steady-state levels were reached by most patients after 3 weeks of continuous oral administration. Sixteen patients elected to undergo FDHT positron emission tomography/computed tomography in the Phase I study. At doses $>120 \mathrm{mg}$, the maximum standardized uptake value $\left(\mathrm{SUV}_{\max }\right)$ average decline at 4 weeks exceeded $90 \%$, while doses $<120 \mathrm{mg}$ demonstrated $\mathrm{SUV}_{\max }$ average decline of $\leq 50 \%$. Eighteen patients $(60 \%)$ had a $\geq 50 \%$ decline in PSA, of which $6 / 18$ had $>90 \%$ decline in PSA at 12 weeks. Median PSA change from baseline at 12 weeks was $-43.2 \%$ (Table 1). The most common adverse events were fatigue, occurring in $47 \%$ of patients, but no study patients experienced a DLT $>$ Grade $3^{19}$ (Table 2 ). The authors reported that although $120 \mathrm{mg}$ seemed to be sufficient to saturate all the ARs, $240 \mathrm{mg}$ in humans was most closely related to the

Table I Summary of major Phase I/II/III studies

\begin{tabular}{|c|c|c|c|c|c|c|}
\hline Study & Phase & $\begin{array}{l}\text { Patients } \\
\text { enrolled }\end{array}$ & Study population & Groups & $\begin{array}{l}\text { PSA reduction at } \\
\text { I } 2 \text { weeks }\end{array}$ & Median TTPP \\
\hline Rathkopf et al ${ }^{19}$ & $\mathrm{I}$ & 30 & $\mathrm{mCRPC}$ & Apalutamide & $-43.20 \%$ & NA \\
\hline \multirow[t]{2}{*}{ Posadas et $\mathrm{al}^{29}$} & $\mathrm{lb}$ & 57 & $\mathrm{mCRPC}$ & Apalutamide/AAP in ENZ naïve & $67 \% \geq 50 \%$ PSA decline & NA \\
\hline & & & & Apalutamide/AAP in ENZ treated & $15 \% \geq 50 \%$ PSA decline & NA \\
\hline Smith et $\mathrm{al}^{20}$ & II & 51 & $\mathrm{~nm}-\mathrm{CRPC}$ & Apalutamide & $-85 \%$ & 24 months \\
\hline \multirow[t]{2}{*}{ Rathkopf et $\mathrm{al}^{21}$} & II & 46 & $\mathrm{mCRPC}$ & Apalutamide in AAP naïve & $-88 \%$ & I8.2 months \\
\hline & & & & Apalutamide in post-AAP & $-22 \%$ & 3.7 months \\
\hline \multirow[t]{2}{*}{ Smith et $\mathrm{al}^{31}$} & III & $\mathrm{I}, 207$ & nm-CRPC (M0 CRPC) & Apalutamide & $-90 \%$ & Not reached \\
\hline & & & & Placebo & $+40 \%$ & 3.7 months \\
\hline
\end{tabular}

Abbreviations: APA, Apalutamide; AAP, Abiraterone Acetate and Prednisone; ENZ, Enzalutamide; NA, not applicable; nm, non-metastatic; mCRPC, metastatic castrationresistant prostate cancer; PSA, prostate-specific antigen; TTPP, time to PSA progression. 
Table 2 Safety profile of apalutamide in Phase I/II studies

\begin{tabular}{lllllll}
\hline Study & Phase & Patients & $\begin{array}{l}\text { Fatigue } \\
\text { (all grades) }\end{array}$ & $\begin{array}{l}\text { Diarrhea } \\
\text { (all grades) }\end{array}$ & $\begin{array}{l}\text { Nausea/anorexia } \\
\text { (all grades) }\end{array}$ & $\begin{array}{l}\text { Back pain } \\
\text { (all grades) }\end{array}$ \\
\hline Rathkopf et al $^{28}$ & I & 9 & $67 \%$ & $56 \%$ & $56 \%$ & $\mathrm{NA}$ \\
Rathkopf et al $^{19}$ & I & 30 & $47 \%$ & $30 \%$ & $30 \%$ & $\mathrm{NA}$ \\
Posadas et al $^{29}$ & Ib & 57 & $42 \%$ & $21 \%$ & $19 \%$ & $22 \%$ \\
Smith et al $^{20}$ & II & 51 & $61 \%$ & $43 \%$ & $39 \%$ & $24 \%$ \\
Rathkopf et al $^{21}$ & II & 46 & $60 \%$ & $44 \%$ & $56 \%$ & \\
\hline
\end{tabular}

Abbreviation: NA, not applicable.

steady-state plasma concentrations associated with tumor regression in the murine xenograft model. ${ }^{11}$ To further test the safety of apalutamide, escalating doses to 300 and $480 \mathrm{mg}$ were tested with adverse events being fatigue, nausea, and abdominal pain, none greater than DLT 3. No seizures were reported in this Phase I study. As such, $240 \mathrm{mg}$ administered orally in a daily fashion was chosen as the formulation for Phase II studies.

\section{Phase II study of apalutamide}

The first Phase II study for apalutamide was performed as a multicenter trial in men with high-risk nonmetastatic CRPC, ${ }^{20}$ defined by the study as either having PSA $\geq 9 \mathrm{ng} / \mathrm{mL}$ or a PSA doubling time $\leq 10$ months. Primary outcome of the study was 12-week PSA response, and secondary outcomes were time to PSA progression (TTPP) and metastasis-free survival (MFS). Patients in the study had no evidence of radiographical metastasis and presented with castrate levels of testosterone ( $\leq 50 \mathrm{ng} / \mathrm{dL})$. Fifty-one patients were enrolled and received continuous daily dosing of apalutamide $240 \mathrm{mg}$ daily until PSA progression, or evidence of clinical progression (skeletal-related event or pain requiring intervention or treatment).

At a median follow-up of 26.9 months, the median PSA reduction from baseline was $85 \%$, with $89 \%$ of patients achieving a $\geq 50 \%$ reduction in PSA (Table 1 ). The median TTPP was 24 months and at the time of analysis, the MFS was not reached. The most common adverse events were fatigue (61\%), diarrhea (43\%), and nausea (39\%) (Table 2). Fifty-three percent of patients with nonmetastatic high-risk CRPC had eventual PSA progression. Notably, no seizures were reported.

\section{Sequencing apalutamide}

In a separate Phase II study, the efficacy of apalutamide before or after treatment with abiraterone/prednisone (AAP) was studied in two cohorts of mCRPC patients: men naïve to AAP (25 patients) vs men who were treated for at least 6 months with AAP (21 patients). ${ }^{21}$ PSA response was observed in $88 \%$ of patients in the AAP-naïve group at 12 -weeks vs only $22 \%$ in the AAP-experienced group. Median TTPP was 18.2 months in the AAP-naïve group vs 3.7 months in the AAP-experienced group (Table 1). Although the median TTPP was only 3.7 months in the AAP-experienced group, the study noted that $43 \%$ of these patients were treated with apalutamide for 6 months or longer, suggesting that $\mathrm{mCRPC}$ remains a heterogeneous entity, and a certain subset will respond more favorably than others. ${ }^{21}$ Similarly, no seizures were noted in this study.

\section{Mutations affecting apalutamide}

AR mutations have been proposed to explain resistance to first- and second-generation antiandrogens. These include mechanisms such as the expression of DNA mutations to the $A R$ gene, mRNA splice variants (AR-V7), and alterations in signaling pathways has also been suggested. ${ }^{22}$ Two main types of AR mutations have been identified: mutations that alter the AR-ligand-binding site to convert antagonist-to-agonist activity, and mutations that result in an AR that has broad ligand specificity, also known as the "promiscuous" receptor. ${ }^{23}$

To further characterize possible mechanisms of resistance to second-generation antiandrogens, LNCaP cell strains chronically treated with enzalutamide and apalutamide were selected for further study. ${ }^{24} \mathrm{~A}$ missense mutation within the AR-ligand-binding domain (at F876L) has been identified in these cells which converts second-generation antiandrogens' antagonistic activity to agonist activity. In vivo studies were subsequently performed, via injection of $\mathrm{LNCaP}$ cell lines expressing the F876L missense mutation into castrate immunodeficient mice. Administration of enzalutamide or apalutamide at concentrations of $30 \mathrm{mg} / \mathrm{kg} / \mathrm{d}$ failed to inhibit growth of the LNCaP tumors. ${ }^{24}$ As with any xenographic model, introduction of mutated cells could potentially change the biology of the disease. Previous studies with LNCaP-AR cells overexpressing AR introduced into immunodeficient mice have shown expected results, ${ }^{11}$ suggesting that xenographic mice models were sufficient for studying the disease and it was not necessary to use mice bred specifically with gene overexpression models. 
Table 3 Resistance profiles to second-generation antiandrogens

\begin{tabular}{llll}
\hline Mutation & $\begin{array}{l}\text { Resistance } \\
\text { profile }\end{array}$ & $\begin{array}{l}\text { Found in } \\
\text { baseline (\%) }\end{array}$ & $\begin{array}{l}\text { Acquired after } \\
\text { treatment (\%) }\end{array}$ \\
\hline F876L/F877L (AR) & $\begin{array}{l}\text { Resistance to } \\
\text { enzalutamide/ } \\
\text { apalutamide }\end{array}$ & $2 / 93(2.2 \%)$ & $3 / 82(3.7 \%)$ \\
T878A (AR) & $\begin{array}{l}\text { Resistance to } \\
\text { abiraterone }\end{array}$ & $3 / 93(3.2 \%)$ & $1 / 82(1.2 \%)$ \\
\hline
\end{tabular}

Note: Data from Rathkopf et al. ${ }^{27}$

Abbreviation: AR, androgen receptor.

To better delineate the acquired nature of the missense mutations, the plasma of 29 patients initially enrolled in the Phase I study of apalutamide ${ }^{19}$ were analyzed with polymerase chain reaction BEAM (Beads, emulsions, amplification and magnetics) technology. Three of 29 patients treated with apalutamide were found to have the F876L mutation after treatment. Pretreatment samples failed to detect the F876L mutation, suggesting that the F876L mutation was selected for and contributes to resistance to second-generation antiandrogens. The AR T878A missense mutation has been associated with resistance to abiraterone ${ }^{25}$ via progesterone activation of mutant AR. ${ }^{26}$

Blood samples of patients who underwent the Phase II apalutamide study were analyzed via BEAM technology to determine the baseline and acquired rates of mutations. ${ }^{27}$ Two of 93 patients analyzed (2.2\%) had the F876L mutation at baseline, and an additional three patients acquired the mutation after treatment (3.7\%). Three of 93 (3.2\%) patients had the T878A mutation at baseline; however, all three patients were from the cohort previously treated with AAP. Two of these patients did respond to ARN-509, with one patient eventually losing the mutation at TTPP on apalutamide. ${ }^{27}$ There were no T878A mutations in the AAP or nonmetastatic CRPC (Table 3). Based on the low baseline and acquired rates of both F876L and T878A mutations, these are unlikely to have played a major or dominant role in the resistance to apalutamide in patients in Phase II studies. ${ }^{27}$

\section{Apalutamide combinations}

The synergistic effects of apalutamide and everolimus (mTOR inhibitors) have been proposed based on xenograft PTEN-deficient CRPC xenograft murine models in mice. Initial studies of nine patients were evaluated with a combination of apalutamide ( $240 \mathrm{mg}$ ) and everolimus (5 and $10 \mathrm{mg}$ ) with prior exposure to AAP in a Phase I study to determine safety, pharmacokinetics, and recommended Phase II dose..$^{28}$ Common adverse events included fatigue, diarrhea, and anorexia (Table 2). The greatest DLT was a grade 3 rash.
Three of nine patients progressed on treatment. One patient remained in the study for over 51 weeks with a $>50 \%$ reduction in PSA. This study was closed before expansion to better evaluate novel PI3K inhibitors. ${ }^{28}$

Although a Phase II study of apalutamide in the post-AAP setting has been reported, a Phase Ib study of apalutamide with AAP was conducted to evaluate the safety and efficacy of this combination. ${ }^{29}$ Fifty-seven patients were separated into two cohorts: abiraterone- and enzalutamide-naïve patients $(\mathrm{n}=18)$ and AA/enzalutamide-treated patients $(\mathrm{n}=39)$. Sixtyseven percent in the naïve group had PSA reduction $>50 \%$ vs $15 \%$ in the AA/enzalutamide-treated group (Table 1). Fatigue, diarrhea, and nausea/vomiting were the most common adverse effects (Table 2). One patient experienced grade 3 DLT fatigue.

\section{SPARTAN trial: apalutamide for the treatment of MO-CRPC}

SPARTAN, a Phase III multicenter, double-blinded, placebo-controlled clinical trial, randomized 1,207 men with nonmetastatic castration-resistant prostate cancer (M0 CRPC) at high risk of disease progression, in a 2:1 fashion, to receive either apalutamide $240 \mathrm{mg}$ PO daily with ADT or placebo with $\mathrm{ADT} .{ }^{30}$ Eligibility to the study required the absence of radiographic evidence of metastasis, as well as a PSA doubling time of $<10$ months despite castrate levels of testosterone, either through continuous use of ADT or having undergone bilateral orchiectomy. Patients were stratified based on presence of local disease, use of osteoporosissparing agents, and PSA doubling time.

The study met its primary endpoint of MFS, with apalutamide demonstrating significant improvement over placebo (40.5 vs 16.2 months, hazard ratio (HR): $0.28,95 \%$ confidence interval (CI): $0.23-0.35, p<0.001)$. Secondary endpoint of time to symptomatic progression was also significantly longer with apalutamide than with placebo (HR: $0.45,95 \%$ CI: $0.32-0.63, p<0.001) .{ }^{31}$ As compared to placebo, apalutamide was associated with a higher rate of rash $(23.8 \%$ vs $5.5 \%)$, hypothyroidism ( $8.1 \%$ vs $2.0 \%)$, and fracture $(11.7 \%$ vs $6.5 \%)$. Only two cases of seizure were noted in the apalutamide-treated cohort. Based on the strength of these trial results, apalutamide received FDA approval in the management of M0 CRPC.

Of note, PROSPER, a randomized Phase III trial of similar design using enzalutamide in M0 CRPC, was run in parallel with SPARTAN, and also showed comparable MFS benefits (36.6 vs 14.7 months, HR: $0.29,95 \%$ CI: $0.24-0.35$, $p<0.0001){ }^{32}$ 
Longitudinal follow-up of the SPARTAN trial will help to answer whether there is an overall survival benefit associated with the use of apalutamide in M0 CRPC, although current data look promising (not reached vs 39.0, HR: 0.7, 95\% CI: $0.47-1.04, p=0.07) .{ }^{31}$ Additionally, further maturation of the exploratory endpoint of second progression-free survival (not reached vs 39.0 months, HR: 0.49), ${ }^{31}$ may yield important insights into optimal sequencing of CRPC agents.

\section{Conclusion}

Current therapeutic agents approved for mCRPC are each associated with median overall survival improvements of three-to-four months, and as such, there remains a continued need to develop new and innovative treatments for mCRPC. Second-generation antiandrogens already play a vital role in the current treatment landscape of CRPC, but improvements can be made. Apalutamide is a novel antiandrogen that has been recently approved for men with nonmetastatic CRPC; its ability to improve MFS and time to symptomatic progression were demonstrated in the Phase III SPARTAN study. Resistance to newer therapeutic agents will inevitably arise, and potential mechanisms of resistance for apalutamide are already being delineated, specifically AR mutations. Both continued follow-up of SPARTAN and additional studies are needed to better understand how apalutamide will make the largest impact in the management of CRPC in terms of sequencing and potential use in drug combinations.

\section{Disclosure}

Dr William Oh is a paid consultant for Astella, Janssen and Pfizer. The authors report no other conflicts of interest in this work.

\section{References}

1. Siegal RL, Miller KD, Jemal A. Cancer statistics, 2016. CA Cancer J Clin. 2016;66(1):7-30.

2. Beer TM, Armstrong AJ, Rathkopf DE, et al. Enzalutamide in metastatic prostate cancer before chemotherapy. $N$ Engl J Med. 2014;371(5): 424-433.

3. Scher HI, Sawyers CL. Biology of progressive, castration-resistant prostate cancer: directed therapies targeting the androgen-receptor signaling axis. J Clin Oncol. 2005;23(32):8253-8261.

4. Lubahn DB, Joseph DR, Sullivan PM, Williard HF, French FS, Wilson EM. Cloning of human androgen receptor complementary DNA and localization to the X chromosome. Science. 1988;240(4850):327-330.

5. Gelmann EP. Molecular biology of the androgen receptor. J Clin Oncol. 2002;20(13):3001-3015.

6. Vis AN, Schroder FH. Key targets of hormonal treatment of prostate cancer. Part 2: the androgen receptor and 5alpha-reductase. BJU Int. 2009;104(9):1191-1197.

7. Edwards J, Bartlett JM. The androgen receptor and signal-transduction pathways in hormone-refractory prostate cancer. Part 1: Modifications to the androgen receptor. BJU Int. 2005;95(9):1320-1326.
8. Edwards J, Bartlett JM. The androgen receptor and signal-transduction pathways in hormone refractory prostate cancer. Part 2: Androgen-receptor cofactors and bypass pathways. BJU Int. 2005;95(9): 1327-1335.

9. Huggins C, Hodges CV. Studies on Prostatic Cancer. I. The effect of castration, of estrogen and of androgen injection on serum phosphatases in metastatic carcinoma of the prostate. Cancer Res. 1941;1: 293-297.

10. Jung ME, Ouk S, Yoo D, et al. Structure-activity relationship for thiohydantoin androgen receptor antagonists for Castration-Resistant Prostate Cancer (CRPC). J Med Chem. 2010;53(7):2779-2796.

11. Clegg NJ, Wongvipat J, Joseph JD, et al. ARN-509: a novel antiandrogen for prostate cancer treatment. Cancer Res. 2011;72(6):1494-1503.

12. Shore ND, Chowdhury S, Villers A, et al. Efficacy and safety of enzalutamide versus bicalutamide for patients with metastatic prostate cancer (TERRAIN): a randomized, double-blind, phase 2 study. Lancet Oncol. 2016;17(2):153-163.

13. Tran C, Ouk S, Clegg NJ, et al. Development of a second-generation antiandrogen for treatment of advanced prostate cancer. Science. 2009; 324(5928):787-790.

14. von Klot CA, Kuczyk MA, Merseburger AS. No androgen withdrawal syndrome for enzalutamide: a report of disease dynamics in the postchemotherapy setting. Eur Urol. 2014;65(1):258-259.

15. Schrader AJ, Schrader MG, Cronauer MV. Re: Christoph A. Von Klot, Markus A. Kuczyk, Axel S. Merseburger. No androgen withdrawal syndrome for enzalutamide: a report of disease dynamics in the postchemotherapy setting. Eur Urol. 2014;65:258-259: Enzalutamide withdrawal syndrome: fact or fiction? Eur Urol. 2014;65(2):e22-e23.

16. Scher HI, Fizazi K, Saad F, et al. Increased survival with enzalutamide in prostate cancer after chemotherapy. $N$ Engl J Med. 2012;367(13): 1187-1197.

17. Graff JN, Gordon MJ, Beer TM. Safety and effectiveness of enzalutamide in men with metastatic, castration-resistant prostate cancer. Expert Opin Pharmacother. 2015;16(5):749-754.

18. Foster WR, Car BD, Shi H, et al. Drug safety is a barrier to the discovery and development of new androgen receptor antagonists. Prostate. 2011;71(5):480-488.

19. Rathkopf DE, Morris MJ, Fox JJ, et al. Phase I study of ARN-509, a novel antiandrogen, in the treatment of castration-resistant prostate cancer. J Clin Oncol. 2013;31(28):3525-3530.

20. Smith MR, Antonarakis ES, Ryan CJ, et al. Phase 2 Study of the safety and antitumor activity of Apalutamide (ARN-509), a potent androgen receptor antagonist, in the high-risk nonmetastatic castration-resistant prostate cancer cohort. Eur Urol. 2016;70(6):963-970.

21. Rathkopf DE, Antonarakis ES, Shore ND, et al. Safety and antitumor activity of apalutamide (ARN-509) in metastatic castration-resistant prostate cancer with and without prior abiraterone acetate and prednisone. Clin Cancer Res. 2017;23(14):3544-3551.

22. Antonarakis ES, Lu C, Wang H, et al. AR-V7 and resistance to enzalutamide and abiraterone in prostate cancer. $N$ Engl J Med. 2014; 371(11):1028-1038.

23. Hara T, Miyazaki J, Araki H, Yamaoka M, Kanzaki N, Kusaka M. Novel mutations of androgen receptor: a possible mechanism of bicalutamide withdrawal syndrome. Cancer Res. 2003;63(1):149-153.

24. Joseph JD, Lu N, Qian J, et al. A clinically relevant androgen receptor mutation confers resistance to second-generation antiandrogens enzalutamide and ARN-509. Cancer Discov. 2013;3(9):1020-1029.

25. Cai C, Chen S, Ng P, et al. Intratumoral de novo steroid synthesis activates androgen receptor in castration-resistant prostate cancer and is upregulated by treatment with CYP17A1 inhibitors. Cancer Res. 2011;71(20):6503-6513.

26. Chen EJ, Sowalsky AG, Gao S, et al. Abiraterone treatment in castrationresistant prostate cancer selects for progesterone responsive mutant androgen receptors. Clin Cancer Res. 2015;21(6):1273-1280.

27. Rathkopf DE, Smith MR, Ryan CJ. Androgen receptor mutations in patients with castration-resistant prostate cancer treated with apalutamide. Ann Oncol. 2017;28(9):2264-2271. 
28. Rathkopf DE, Slovein SF, Morris MJ, Danila DC, Delacrus A, Shelkey G. Targeting reciprocal feedback inhibition: Apalutamide and everolimus in patients with metastatic castration-resistant prostate cancer (mCRPC) [abstract]. J Clin Oncol. 2017;35(6_suppl):204.

29. Posadas EM, Chi KN, De Wit R, De Jonge MJ, Gerhardt A, Friedlander TW. Phase Ib study of apalutamide (APA) with abiraterone acetate (AA) and prednisone $(\mathrm{P})$ in patients (pts) with metastatic castration-resistant prostate cancer (mCRPC): Update on safety and efficacy [abstract]. J Clin Oncol. 2017;35(6_suppl):135.

30. Smith MR, Liu G, Shreeve SM, Mathey S, Sosa A, Kheoh TS. A randomized double-blind, comparative study of ARN-509 plus androgen deprivation therapy (ADT) versus ADT alone in nonmetastatic castration-resistant prostate cancer (M0-CRPC): The SPARTAN trial. $J$ Clin Oncol. 32:(15_suppl):TPS5100.
31. Smith MR, Saad F, Chowdhury S, et al. Apalutamide treatment and metastasis-free survival in prostate cancer. N Engl J Med. Epub 2018 Feb 8.

32. Hussain M, Fizazi K, Saad F, et al. PROSPER, a phase III, randomized, double blind, placebo (PBO)-controlled study of enzalutamide (ENZA)in men with nonmetastatic castration-resistant prostate cancer (M0 CRPC) [abstract]. J Clin Oncol. 2018;36 (suppl 6S; abstr 3).

\section{Publish your work in this journal}

OncoTargets and Therapy is an international, peer-reviewed, open access journal focusing on the pathological basis of all cancers, potential targets for therapy and treatment protocols employed to improve the management of cancer patients. The journal also focuses on the impact of management programs and new therapeutic agents and protocols on

\section{Dovepress}

patient perspectives such as quality of life, adherence and satisfaction. The manuscript management system is completely online and includes a very quick and fair peer-review system, which is all easy to use. Visit http://www.dovepress.com/testimonials.php to read real quotes from published authors.

Submit your manuscript here: http://www.dovepress.com/oncotargets-and-therapy-journal 